\title{
LA CALIDAD EN EDUCACIÓN SUPERIOR
}

\author{
FLORENTINO MONSALVE MURILLO *
}

\section{EL CONTEXTO DE LA CALIDAD}

En los últimos 30 años, la sociedad colombiana encabezada por el Estado ha asumido como prioritaria la estrategia del mejoramiento de la calidad de la educación porque se considera que esta es una condición necesaria para la modernización de los procesos productivos.

Desde la reforma educativa de 1960 , pasando por el Decreto 080 de 1980 hasta la Ley 30 de 1992 y las normas que la reglamentan y la Ley 115 de 1993, ha habido una evidente preocupación del estado por modernizar el sistema educativo colombiano.

Sin embargo, la modernidad, entendida como el dominio de la razón sobre los sentimientos en la decisión del qué hacer y cómo hacerlo, sigue siendo una aspiración lejana. Las decisiones se toman atendiendo a intereses individuales inmediatos y no orientadas por el conocimiento y la previsión de las consecuencias.

Las instituciones de Educación Superior no escapan a este escenario. La autonomía consagrada en la Ley 30 como un derecho y un deber consustancial de las universidades, requiere madurez en las instituciones para ejercerla con eficiencia, eficacia y responsa-

- Profesor Titular de la Universidad Surcolombiana. bilidad. La mayoría de las instituciones no poseen el mínimo grado de madurez que garantice el ejercicio correcto de la autonomía y en consecuencia abusan de ella.

La proliferación de programas que se crean a diario sin la existencia de mínimos requisitos de infraestructura fisica, académica y cultural en ciudades y pueblos; la proliferación de especializaciones que no especializan en nada y de magísteres que no forman investigadores en ninguna ciencia, que se realizan en unos cuantos fines de semana y gradúan a los estudiantes independientemente de su desempeño académico, son apenas muestra de lo que ocurre.

El Sistema Nacional de Acreditación, establecido por la Ley 30, pretende resolver este problema, pero por ser voluntario, no afectará a las instituciones de baja calidad quienes continuarán formando, profesionales, magisteres y especialistas que compiten con los formados por las universidades de buena calidad en el mercado laboral. Se supone que los estudiantes seleccionarán las universidades acreditadas por su calidad, pero, puesto que en la sociedad no se privilegia el saber sino los títulos y las relaciones interpersonales o gremiales, muchos estudiantes preferirán las instituciones que les ofrezcan títulos con esfuerzos mínimos; lo anterior ocurrirá especialmente en los postgrados, a los que acceden los estudiantes motivados por la necesidad de ascender en 
el escalafón laboral y no porque sus funciones requieran conocimientos especializados o avanzados. La mayoría de los postgraduados continúan haciendo lo mismo y de la misma manera.

\section{EL CONCEPTO DE CALIDAD}

El concepto de calidad referido a la educación superior ha sido, en los últimos años, objeto de debate en todo el mundo, y particularmente en Colombia, especialmente a partir de la Constitución de 1991 y de la Ley 30 de 1992, que ordenan la organización de un sistema de acreditación como mecanismo de fomento a la calidad de la educación superior. ${ }^{1}$

Diferentes autores utilizan diferentes conceptos de calidad; generalmente, al intentar decir lo que es la calidad, se limitan a describir los sujetos que la poseen sin detenerse a concretar su contenido esencial. El C.N.A. afirma:

"La calidad se entiende como un atributo integral de algo, resultado de una síntesis de los componentes y los procesos que lo producen y distinguen... La calidad de algo es aquello que le corresponde necesariamente y que al faltarle afecta su naturaleza, su ser propio." (CNA, junio de 1996)

La presencia de diversidad de intereses en la educación superior hace que existan diferentes perspectivas acerca de lo que es la calidad, pero prevalecen las perspectivas de la eficiencia, la excelencia y la eficacia.

La calidad es un constructo social, y la definición de calidad que prevalece en cualquier contexto dado, es la que recibe el apo- yo de los más poderosos. Debido a que la calidad es una construcción social, nunca carece de valor. Siempre se asocia con lo que es bueno y valioso.

Desde la perspectiva gubernamental, la calidad en la educación superior está estrechamente relacionada con la noción de control, a juzgar por los términos utilizados para referirse a ella: Responsabilidad de la calidad, evaluación de calidad, garantía de calidad, control de calidad. Lo anterior obedece al interés de los gobiernos por controlar el gasto en educación superior. ${ }^{2}$

En el mundo de la industria y el comercio, el concepto de calidad refleja un compromiso con la filosofía del mercado, y una creencia de que los mercados son los mejores medios para la producción y distribución de productos, incluyendo los servicios edu. cativos.

En sus formas más avanzadas, el control de calidad de la educación superior incluye la creación de mercados en los cuales las instituciones se constituyen como compañías privadas, donde el gobierno y otras empresas no gubernamentales compran cupos y las instituciones tienen que competir unas contra otras por venderlos. Las que no lo. gren los estándares de calidad exigidos por los compradores tendrán que salir del mercado.

Según Goodlad, en este contexto, la ca. lidad es una medida de eficiencia. ${ }^{3}$

Quienes enfocan la calidad desde la perspectiva de la excelencia asumen la calidad como el atributo que hace a algo excepcional. El C.N.A, en el documento Lineamientos

1. C.N.A (Consejo Nacional de Acreditación). Lineamientos para la Acreditación. Segunda Edición Santa Fe de Bogotá, junio de 1996.

2. Brook L. y Nonhebel D. (1996). La Calidad y el Desarrollo de Indicadores de Impacto Social en Educación Superior. Ascun, Santa Fe de Bogotá.

3. Goodlad S (1995). The Quest for Quality. SRHE/OU Press Buckingham. 
para la acreditación de programas e instituciones de Educación Superior dice:

"La calidad de algo es la medida en que ese algo se aproxima al prototipo ideal definido históricamente como realización óptima de lo que le es propio según el género a que pertenece. ...Alude tanto a la posibilidad de distinguir un miembro de los demás del género como a la distancia entre cada uno de los miembros al prototipo definido para ese género. Así se puede hablar de un mayor o menor grado de calidad efectiva..." (CNA, junio de 1996)

"Calidad, es el conjunto de cualidades que tiende a lograr una universidad que está en búsqueda de la excelencia." (Documento de las 12 universidades, Febrero 1996).

La calidad vista como excelencia se asocia con altos niveles de desempeño y rendimiento. Se supone que los estándares son objetivos, universales y estáticos; cuando, en la realidad, los estándares son concertados dentro de contextos particulares.

En Colombia, la perspectiva de la excelencia está estrechamente ligada con las universidades Nacional, Valle. Antioquia, UIS, Andes y Javeriana. Estas son las Instituciones modelo, cuyo paradigma debe ser seguido por las otras que para ser de buena calidad deben ser como ellas. Estas a su vez deben acercarse a ser como los modelos mundiales: Oxford, Cambridge, Lovaina, Harvard, París, Roma, Salamanca etc.

La perspectiva de la excelencia es altamente elitista: La calidad no está sujeta a una definición explícita, sino que es un espacio controlado por la elite y accesible únicamente en circunstancias muy especiales. La superioridad social y la exclusión están dentro de los valores que sustentan tal con- cepto, y no es sorprendente que sea el enfoque más frecuentemente usado en las discusiones contra la ampliación del acceso a la educación superior. " Un incremento en la participación amenaza la calidad." Los excelentes para serlo, tienen que ser necesariamente pocos.$-^{4}$

Al asumir la calidad desde la perspectiva de la eficacia se la entiende como correspondencia con el propósito y se supone que un objeto exhibe calidad si desempeña la función para la cual está diseñado.

“...la evaluación deberá incluir no sólo aspectos académicos....sino cómo se encarnan en ellos la misión y el proyecto educativo institucional..." (Documento de las 12 universidades, Febrero 1996).

“ ... Un factor de calidad que también debe evaluarse, en términos de la distancia a un prototipo, es la mayor o menor diferencia entre lo que una institución es y lo que ella ha definido como su misión que es parte sustancial de su identidad." (CNA junio de 1996)

"El concepto de calidad como correspondencia con el propósito, diverge del concepto de calidad como algo especial, distinto, elitista, otorgador de estatus o difícil de conseguir, porque define la calidad funcionalmente". (Harvey 1993).

La perspectiva de correspondencia con el propósito, no pretende que la calidad sea absoluta y por eso no agota la discusión sobre lo que queremos lograr. Reconoce la importancia de los propósitos del estado, la sociedad, los profesores, los estudiantes y los funcionarios en la definición de calidad. No limita la reflexión y discusión sobre valores y prioridades. Es un proceso abierto: la misión es pública y disponible al pú-

4. Balla J, D Neubauer and L Gow, (1995). Managing Higher Education. In Journal of Institutional Research in Australasia. 
blico para su escrutinio. Además se relaciona fuertemente con los enfoques usados comercialmente, lo que la hace políticamente aceptable. Y tal vez lo más pertinente, puede aplicarse a la compleja variedad de instituciones.

\section{LA EVALUACION DE LA CALIDAD EN EDUCACION SUPERIOR}

Las razones para evaluar la calidad en la educación superior son múltiples:

Por una parte, el Sistema Nacional de Acreditación, ha sido creado por la Constitución con el objeto de "garantizar a la Sociedad que las instituciones de educación superior cumplan los más altos requisitos de calidad y que realizan sus propósitos y objetivos". Este mandado obliga a las instituciones a desarrollar estrategias y mecanismos que les permitan poner en evidencia ante el estado y la sociedad la calidad con que asumen los procesos educativos. La evaluación de la calidad es el elemento nuclear de todo proceso de acreditación. ${ }^{5}$

Por otra parte, sólo las organizaciones que mejoran permanentemente su calidad están llamadas a sobrevivir en las condiciones cambiantes cada vez más complejas, en que se desarrolla la producción y difusión del conocimiento como estrategias surgidas de consensos amplios entre los diferentes actores sociales. Se exige a las universidades centrar su acción en la búsqueda de mejores resultados en la formación de recursos humanos y de capacitación para el manejo racional de los nuevos conocimientos científicos y tecnológicos, y se les conmina a romper su aislamiento e introducir modalidades de acción que abran a los actores mejores espacios de autonomía en las decisiones, asi como mayor responsabilidad por los resultados. 6

La educación superior, debe centrarse en la formación integral del ser humano como individuo capaz de asimilar, transformar y crear conocimiento científico, tecnológico y humanístico y asumir una postura crítica y autónoma frente al conocimiento. La sociedad moderna requiere individuos capaces de enfrentar autónomamente los conocimientos, la ciencia, los saberes, la técnica y las responsabilidades éticas en armonía con sigo mismo y con la realidad económica y social. ?

Los trabajadores del conocimiento son los llamados a dinamizar los procesos productivos. Los computadores y máquinas automáticas, ya están haciendo casi todo lo que saben hacer los ingenieros y técnicos formados en las universidades. La instrucción en el manejo de procesos y procedimientos, en la aplicación de fórmulas matemáticas, y otras actividades similares, deben dar paso a la formación del pensamiento, a la apropiación de los fundamentos de las ciencias, a la comprensión de la problemática social del hombre y de la sociedad y a la estructuración de propuestas creativas para resolverla. ${ }^{8}$

El crecimiento acelerado del conocimiento científico, exige profesionales capaces de aprender permanentemente, de seleccionar información pertinente, de utilizar con eficacia las tecnologías de la informática y la comunicación. Más temprano que tarde, los títulos que expide la universi-

5. Lineamientos para la Acreditación. Consejo Nacional de Acreditación 1.996.

6. Salto Social Educativo, Presidencia de la República, 1995.

7. Misión para la Modernización de la Universidad Pública Informe Final

8. TOFFLER, A. El cambio de poder. Plaza y Janés, 1992. 
dad, tendrán carácter de certificados que acreditan a los individuos como capaces de seguir aprendiendo en el futuro. ${ }^{9}$

Las universidades que sobrevivan en el próximo siglo, serán las que logren flexibilizar sus estructuras organizacionales y sus métodos de trabajo para adecuar permanentemente su acción a la definición del futuro que ellas mismas se han planteado. Las universidades, deberán convertirse en organizaciones que aprenden, como entidades en continua transformación que no solo responden sino que pueden adelantarse a la realidad. Para lograr lo anterior tendrán que construir, metodologías que permitan mostrar objetivamente el grado de calidad con que realizan sus compromisos con la ciencia, la técnica, el hombre y la sociedad. ${ }^{10}$

El proceso de evaluación de la calidad tiene connotaciones estratégicas:

La teoria sobre administración de organizaciones incorpora el concepto de estrategia, que consiste en la articulación de los procesos de toma de decisiones que comprometen a todos los niveles y unidades de la institución.

La estrategia compromete así, a todos los niveles jerárquicos de la organización: Corporativo (Consejos Superior y Académico, Rector y Vicerrectores), Funcional, (Consejos de Facultad, Decanos, y Jefes de Divisiones Administrativas) y Operativo (Programas, Departamentos, Institutos, Centros, Unidades Administrativas), definiendo las responsabilidades académicas, administrativas y asistenciales correspondientes a todas las personas involucradas en el sistema decisional.
Para lograr los propósitos de una estrategia para el cambio, es determinante la existencia de una cultura institucional, entendida como "el conjunto de supuestos básicos y creencias, profundamente arraigados y compartidos por todos los miembros de la organización que influyen y condicionan significativamente los comportamientos individuales"."1

Toda evaluación tiene dos dimensiones básicas: la dimensión intrínseca y la extrínseca. La primera corresponde al grado de concordancia de los resultados de las operaciones que la organización realiza con los propósitos previamente explícitos y definidos, por ella misma, para tales operaciones. La segunda corresponde al grado de concordancia de los resultados globales de la operación de la organización con las expectativas e intereses de los sectores externos involucrados (gobierno y sociedad civil).

Tanto la dimensión intrínseca como la extrínseca de la evaluación deben ser revisadas continua, sistemática y organizadamente basándose en hechos y opiniones de los afectados por la organización y de manera integral, es decir articulada con los procesos de planeación, presupuestación y acreditación como mejoramiento de la calidad. ${ }^{12}$

\section{EL PROCESO DE AUTOEVALUACION DE LA CALIDAD}

La autoevaluación consiste en un proceso de revisión continua, sistemática y organizada que hace la misma universidad de sí misma, o de alguno de sus componentes de manera integral y participativa que, confi-

9. Combs, P. Universidad e investigación. Asterisco Cultural No 4. Madrid 1991

10. Pabón F. Nohra. Autoevaluación Institucional. Cuadernos ASCUN.Acreditación .

11. Ayarza E. Hernán. El Proceso de Autoevaluación. Cuadernos ASCUN.Acreditación.

12 Idem. Página 29. 
gura un sistema eficiente y eficaz de información, basado en hechos y opiniones de los integrantes de la organización y que da cuenta de la concordancia de las acciones planificadas y ejecutadas con los propósitos para las cuales fueron diseñadas. ${ }^{13}$

El proceso de autoevaluación tiene por objetivo el estudio del desempeño de la institución y de sus unidades, organizado y conducido por ella misma, en la perspectiva de su propio proyecto educativo, para determinar el grado en que la institución y sus unidades están logrando sus propósitos previamente establecidos e identificar los problemas que están dificultando su excelente realización. Este estudio, integrado por elementos descriptivos, analíticos y reflexivos, con predominio de los últimos, supone un compromiso con el cambio y con el mejora- miento de la calidad institucional. En consecuencia no debe quedarse en un simple diagnóstico explicativo de las debilidades y fortalezas sino que debe plantear estrategias viables para la superación de las primeras utilizando eficaz y eficientemente las segundas. La autoevaluación es la fuente renovadora de la agenda institucional para el mejoramiento de la calidad. ${ }^{14}$

Este proceso es esencialmente técnico, aunque involucra decisión política de las autoridades institucionales. Su efectividad está íntimamente relacionada con la capacidad de la organización para identificar y explicar problemas, buscar soluciones y emplear estrategias para introducir, administrar y sustentar los cambios requeridos. Su propósito principal es el mejoramiento del hacer institucional.

\section{BIBLIOGRAFIA}

1. Orozco Silva, Luis Enrique (1993). Reestructuración de la Educación Superior Colombiana 1991-1993. TEXTOS/M.D.U./27. Universidad de Los Andes Santafé de Bogotá 1993.

2. Brook L. y Nonhebel D. (1996). La Calidad y el Desarrollo de Indicadores de Impacto Social en Educación Superior. Ascun, Santa Fe de Bogotá.

3. Balla J, D Neubauer and L Gow, (1995). Managing Higher Education. In Journal of Institutional Research in Australasia.

4. Fogel B R (1977). Design for Change: Higher Education in the Service of Developing Countries. International Council for Educational Development New York.

5. Harvey L and Greren D. (1983). Defining Cuality in Assessment and Evaluation in Higher Education. 18(1).

6. C.N.A (Consejo Nacional de Acreditación). Lineamientos para la Acreditación. Segunda Edición Santa Fe de Bogotá, junio de 1996.

7. Grupo de las 12 Universidades. Caracteristicas de Calidad: Hacia una Universidad Autorregulada. Ascún Colombia, Febrero de 1996.

8. Goodlad S (1995). The Quest for Quality. SRHE/OU Press Buckingham.

13. Autoevaluación para Instituciones de Educación Superior, Pautas y Procedimientos, CINDE, 1994.

14. TOBARGA T. Huáscar. Balance de la autoevaluación de las universidades públicas en México. Cuadernos ASCUN. 